\title{
Physiological Fluid Specific Agglomeration Patterns Diminish Gold Nanorod Photothermal Characteristics
}

\author{
Kristen K. Comfort ${ }^{1,2}$, Jared W. Speltz ${ }^{3}$, Bradley M. Stacy ${ }^{1,2}$, \\ Larry R. Dosser ${ }^{3}$, Saber M. Hussain ${ }^{\text {* }}$ \\ ${ }^{1}$ Molecular Bioeffects Branch, Human Effectiveness Directorate, Air Force Research Laboratory, \\ Wright-Patterson Air Force Base, Dayton, USA \\ ${ }^{2}$ Department of Chemical and Materials Engineering, University of Dayton, Dayton, USA \\ ${ }^{3}$ Mound Laser \& Photonics Center Inc., Miamisburg, USA \\ Email: *saber.hussain@wpafb.af.mil
}

Received August 28, 2013; revised October 3, 2013; accepted October 17, 2013

Copyright (C) 2013 Kristen K. Comfort et al. This is an open access article distributed under the Creative Commons Attribution License, which permits unrestricted use, distribution, and reproduction in any medium, provided the original work is properly cited.

\begin{abstract}
Investigations into the use of gold nanorods (Au-NRs) for biological applications are growing exponentially due to their distinctive physicochemical properties, which make them advantageous over other nanomaterials. Au-NRs are particularly renowned for their plasmonic characteristics, which generate a robust photothermal response when stimulated with light at a wavelength matching their surface plasmon resonance. Numerous reports have explored this nanophotonic phenomenon for temperature driven therapies; however, to date there is a significant knowledge gap pertaining to the kinetic heating profile of Au-NRs within a controlled physiological setting. In the present study, the impact of environmental composition on Au-NR behavior and degree of laser actuated thermal production was assessed. Through acellular evaluation, we identified a loss of photothermal efficiency in biologically relevant fluids and linked this response to excessive particle aggregation and an altered Au-NR spectral profile. Furthermore, to evaluate the potential impact of solution composition on the efficacy of nano-based biological applications, the degree of targeted cellular destruction was ascertained in vitro and was found to be susceptible to fluid-dependent modifications. In summary, this study identified a diminution of Au-NR nanophotonic response in artificial physiological fluids that translated to a loss of application efficiency, pinpointing a critical concern that must be considered to advance in vivo, nano-based bio-applications.
\end{abstract}

Keywords: Gold Nanorod; Nanophotonic; Agglomeration; Artificial Physiological Fluid; Photothermal Application

\section{Introduction}

Due to their unique physicochemical properties, gold nanorods (Au-NRs) are being increasingly utilized in consumer and medical applications, including photothermal therapy, bioimaging, drug delivery, and site specific catalysis [1-3]. Specifically, these distinctive nanogold parameters include a general biocompatibility, plasmonic properties, enhanced surface reactivity, and ease of functionalization [4]. Au-NRs also possess the shapespecific advantages of tunable aspect ratio (AR) and strong longitudinal surface plasmon (LSP) absorbance in the near infrared (NIR) range [4]. Due to the fact that NIR light excites the LSP resonance of the Au-NRs, they possess exceptional photothermal capabilities, converting absorbed light into thermal energy with high efficiency

*Corresponding author.
[5,6]. This nanophotonic characteristic, coupled with the fact that NIR light is minimally absorbed by tissues, makes Au-NRs an ideal candidate for photothermal based therapies and applications.

One recent Au-NR application being explored is the targeted ablation of cancer cells through photothermal therapy [7]. This process is being enhanced through the addition of targeting agents and chemotherapy treatments to the Au-NR surface $[8,9]$. Moreover, the nanophotonic response of Au-NRs has been developed into a delivery mechanism for the systematic release of a target biomolecule, with specific examples including DNA oligonucleotides and the anticancer drug paclitaxel [10]. However, as cellular environments are extremely susceptible to temperature fluxuations, photothermal-based applications have a very narrow temperature range for optimal efficiency, which needs to be considered during the 
design process.

The variance of nanorod optical properties as a function of AR is well defined with the LSP band shifting further into the NIR spectrum with increasing AR [11]. Theoretical models have linked AR dependent spectral signatures of Au-NRs to projected photothermal efficiencies and have identified particle absorbance to be the predominant factor contributing to the nanophotonic response [11]. Furthermore, previous studies have shown that ionic strength and refractive index of the dispersant fluid directly impacts the absorbance, agglomeration state, and stability of Au-NRs: all of which have the potential to modify the innate photothermal abilities of these nano-sized particles [12,13].

Agglomeration is a major concern regarding the physical behavior of nanomaterials, and is a property frequently overlooked. Aggregation of particles has been shown to have wide-reaching effects and influences outcomes including cytotoxicity, rate of internalization, particle distribution, and spectral profiles [12]. Agglomeration induced modifications to a nanomaterial's absorption spectrum would cause a significant change in nanophotonic response as absorbance is the dominant physical basis for photothermal therapy and imaging applications $[14,15]$. Nano-gold has previously demonstrated a high degree of agglomeration in biological systems [16,17], highlighting the necessity to elucidate particle behavior in appropriate physiological environments.

To date, there is a significant lack of information regarding the kinetic heating patterns of nanomaterials solutions in physiological fluids, due to the constraints associated with obtaining in vivo samples. One mechanism to overcome this challenge is the utilization of artificial physiological fluids, which mimic the composition and behavior of a biological fluid. Artificial fluids also allow for the characterization and behavioral analysis of nanomaterials in a physiologically relevant environment with greater ease and reproducibility.

This study evaluated the behavior and photothermal response of Au-NRs in multiple biological environments and linked heating profiles to their respective physical properties. The fluids under examination included artificial alveolar fluid (AF), interstitial fluid (IF), and cerebrospinal fluid (CSF). Composition was found to dictate $\mathrm{Au}-\mathrm{NR}$ agglomeration, independent of AR, thereby modifying the heating kinetics. Moreover, to verify the impact of these heating alterations in vitro, the degree of $\mathrm{Au}-\mathrm{NR}$ induced cellular ablation as a function of fluid composition was ascertained. This work revealed that fluid-induced particle agglomeration in a biological environment can significantly alter the photothermal response of Au-NRs and reduce the efficiency of laser mediated nano-based biological applications.

\section{Experimental}

\subsection{Au-NR Synthesis and Characterization}

The Au-NRs were synthesized through a dual surfactant, wet-chemistry based process utilizing cetyltrimethlyammonium bromide (CTAB) and benzyldimethylammonium chloride (BDAC) as previously described [18]. Synthesis was carried out through the addition of gold seeds to an aqueous solution, after which gold ions were reduced on the seed surface to form nanorods. The aspect ratio was controlled during this process through the initial concentration of BDAC within the growth solution. The high-grade $\mathrm{CTAB}$ and BDAC were purchased from GFC Chemicals and TSI Incorporated, respectively. All other chemicals involved in synthesis were purchased from Sigma Aldrich.

The Au-NRs were purified through a combination of the well-established protocols of centrifugation and depletion-induced separation [19] and functionalized with 5 $\mathrm{kD}$ thiolated polyethylene glycol (PEG) [20]. Following synthesis, the Au-NRs were characterized with transmission electron microscopy (TEM: Hitachi H-7600) for size and morphology, UV-VIS analysis (Varian Cary 5000) for spectral signature, and inductively coupled plasma mass spectrometry (Perkin Elmer ICP-MS 300D) for particle concentration.

\subsection{NIR Laser Exposure}

An $808 \mathrm{~nm}$, continuous wave fiber coupled laser diode (Oclarao Technology Limited) was utilized as the NIR light source. An average laser power of $3.5 \mathrm{~W}$ was delivered and controlled through a Newport laser diode driver. The fiber was paired to an optics system for collimation, producing a power density of $0.774 \mathrm{~W} / \mathrm{cm}^{2}$. The beam path was targeted longitudinally to a cuvette containing 2 $\mathrm{mL}$ of a $25 \mu \mathrm{g} / \mathrm{mL}$ Au-NR solution, which was monitored with a Dostmann electronic P6000 thermocouple positioned within the cuvette, but outside the direct beam path. The reproducibility of this set-up was verified by quantifying the kinetic heat response of the Au-NRs in control media in triplicate with negligible differences observed. For cellular laser irradiation, the diode was aimed in a down position at adherent cells located in a 96 well plate.

\subsection{Thermodynamic Model}

By assuming the heat lost to the environment is negligible in comparison to the quantity of generated energy, the rate of heat production can be quantified by a simplified version of the first law of thermodynamics, as follows.

$$
\dot{Q}=\frac{C_{v} \Delta T}{t}
$$


where $Q$ is the rate of heat generation $(\mathrm{J} / \mathrm{s}), C_{v}$ is the fluid heat capacity, $\Delta T$ is the change in solution temperature, and $t$ is the duration. In this analysis it was assumed that the heat capacity and density of water were unaltered by the presence of Au-NRs and were 4.14 $\mathrm{J} / \mathrm{g}-\mathrm{K}$ and $1 \mathrm{~g} / \mathrm{cm}^{3}$, respectively. When applied for the alternative fluids, the assumption was made that the $\Delta T$ term was dominant over the $C_{v}$ and the heat capacity of the artificial fluids was approximated to be equal to that of water.

\subsection{Nanorod Stability Assessment}

Following dispersion in the denoted artificial fluids, nanorod characterization was performed through darkfied (DF) microscopy, UV-VIS, zeta-potential, and dynamic light scattering (DLS). For DF imaging, a small volume of a $25 \mu \mathrm{g} / \mathrm{mL}$ Au-NR sample was spotted onto a microscope slide and the agglomerates were imaged using a CytoViva 150 attachment on an Olympus BX41 microscope. The zeta potential and DLS analyses, which assess surface charge and agglomerate size, respectively, were performed on a Malvern Zetasizer Nano ZS series. All stability assessments were performed on freshly dispersed Au-NR solutions.

\subsection{Cell Culture and Artificial Physiological Fluids}

Human kertatinocyte, $\mathrm{HaCaT}$, cells were a kind gift from the Army Research Laboratory and were maintained in RPMI 1640 cell culture media (ATCC) supplemented with $10 \%$ heat-inactivated fetal bovine serum and $1 \%$ penicillin-streptomycin. Cells were stored in a humidified incubator controlled at $37^{\circ} \mathrm{C}$ and $5 \% \mathrm{CO}_{2}$. In addition to traditional growth media (GM) this study explored the impact of a number of artificial fluids on Au-NR behavior, including alveolar fluid, interstitial fluid, and cerebrospinal fluid. The artificial fluids were created from previously published recipes [21], and are comprised of a predetermined concentrations of multiple salt solutions (Sigma Aldrich).

\subsection{Cell Viability Measurements}

HaCaTs were seeded into a 96 well plate at a concentration of $2.0 \times 10^{4}$ cells per well and returned to the incubator for 24 hours. The cells were then exposed to the indicated conditions, underwent NIR laser irradiation for 1 minute, then replenished with fresh growth media. The next day viability was assessed using the CellTiter 96 Aqueous One Solution (Promega Corp.) which monitors mitochondrial function through a tetrazolum reagent (MTS), in accordance with the manufacturer's protocol.

\subsection{Statistical Analysis}

Data are expressed as the mean \pm standard error of the mean (SEM). A two way ANOVA analysis was run using Graph Pad Prism followed with a Bonferroni adjustment to determine statistical significance. An asterisk denotes a $\mathrm{p}$-value $<0.05$.

\section{Results and Discussion}

\subsection{Au-NR Characterization}

Au-NRs were successfully and reproducibly synthesized with target ARs of approximately 3.0, 3.5, and 4.0; representative TEM images are shown in Figure 1. All Au-NRs in this study were functionalized with thiolated PEG as it has been shown to augment thermal dissipation from Au-NRs during laser irradiation [22]. In addition, thiolated PEG is capable of displacing the surface bound $\mathrm{CTAB}$ which is required for nanorod synthesis, but has also been shown to be extremely cytotoxic [23]. Prior to experimentation, the efficient removal of CTAB was verified through a viability assessment of the Au-NRs; all of which were found to be biocompatible after a 24 hour exposure at concentrations up to $100 \mu \mathrm{g} / \mathrm{mL}$ (Figure 2).

Following synthesis and functionalization, the Au-NRs underwent extensive characterization to identify their physical parameters prior to investigating their innate nanophotonic potential. The characterization results are summarized in Table 1. TEM images were used to cal-

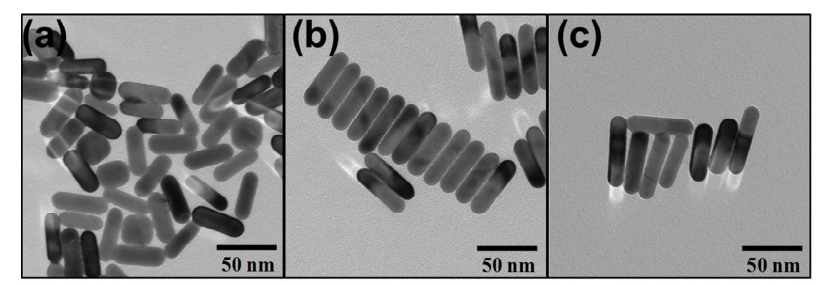

Figure 1. Representative TEM images of gold nanorods following synthesis, purification, and functionalization with PEG. The gold nanorods have defined and uniform aspect ratios of (a) 3.0, (b) 3.5, and (c) 4.0.

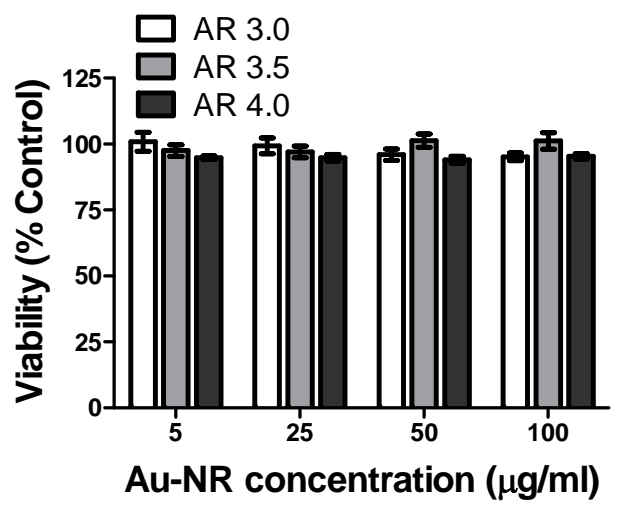

Figure 2. Viability assessment of Au-NRs following functionalization with PEG demonstrated no particle induced cytotoxic effects after 24 hours. 
Table 1. Gold nanorod characterization.

\begin{tabular}{cccc}
\hline AR & Actual AR & Zeta potential $(\mathrm{mV})$ & Agglomerate size $(\mathrm{nm})$ \\
\hline 3.0 & 2.8 & $-23.1 \pm 1.5$ & $61.0 \pm 1.4$ \\
3.5 & 3.6 & $-21.4 \pm 1.3$ & $68.9 \pm 1.2$ \\
4.0 & 3.9 & $-19.3 \pm 0.7$ & $76.6 \pm 1.9$ \\
\hline
\end{tabular}

culate the primary size and verify the rod morphology of the Au-NRs. The width for the nanorods was approximately $15 \mathrm{~nm}$, with a length of 41,54, and $60 \mathrm{~nm}$ for AR $3,3.5$, and 4. Furthermore, these images indicated excellent material parameter uniformity among particles. Zeta potential analysis determined that all the particles displayed a negative surface charge; further confirming that the positively charged CTAB was effectively removed. Agglomerate size was ascertained in media through DLS, and identified a small degree of inter-particle association, which was expected as all nanomaterials agglomerate to some extent in solution. Lastly, the spectral signatures of the Au-NR sets were obtained (Figure 3(a)) and further demonstrated the quality of these particles, which possessed distinct, sharp surface plasmon resonance peaks.

\subsection{Correlating Au-NR Absorbance to the Kinetic Photothermal Response}

As anticipated, UV-VIS analysis of the nanorods demonstrated that with increasing AR the longitudinal peak shifted further into the NIR spectrum (Figure 3(a)). One consequence of this spectral shift is that each Au-NR set possessed a different absorbance at $808 \mathrm{~nm}\left(\mathrm{~A}_{808}\right)$, the operational laser wavelength. Therefore, utilizing AuNRs of varying AR provided a metric to evaluate the correlation between particle absorbance and photothermal efficiency while simultaneously removing other variable such as composition, surface chemistry, and concentration. During NIR laser irradiation, the Au-NRs demonstrated a differential heating response based on their respective $A_{808}$ values (Figure 3(b)). Furthermore there existed a direct proportionality between the $\mathrm{A}_{808}$ of each Au-NR set and degree of observed solution heating.

Next, a simple thermodynamic model was used to evaluate the rate of thermal production $(Q)$ for each AR. This model was implemented for the time frame of 1 - 4 minutes to capture the linear phase of the photothermal response. The results of this analysis are shown in Figure 3(c) and provide a clear, numerical connection between Au-NR absorbance, change in solution temperature, and kinetic heat production.

\subsection{Fluid Dependent Reduction of Photothermal Efficiency}

One factor that has been shown to dictate the nanomaterial physical behavior, including absorbance values, sur-
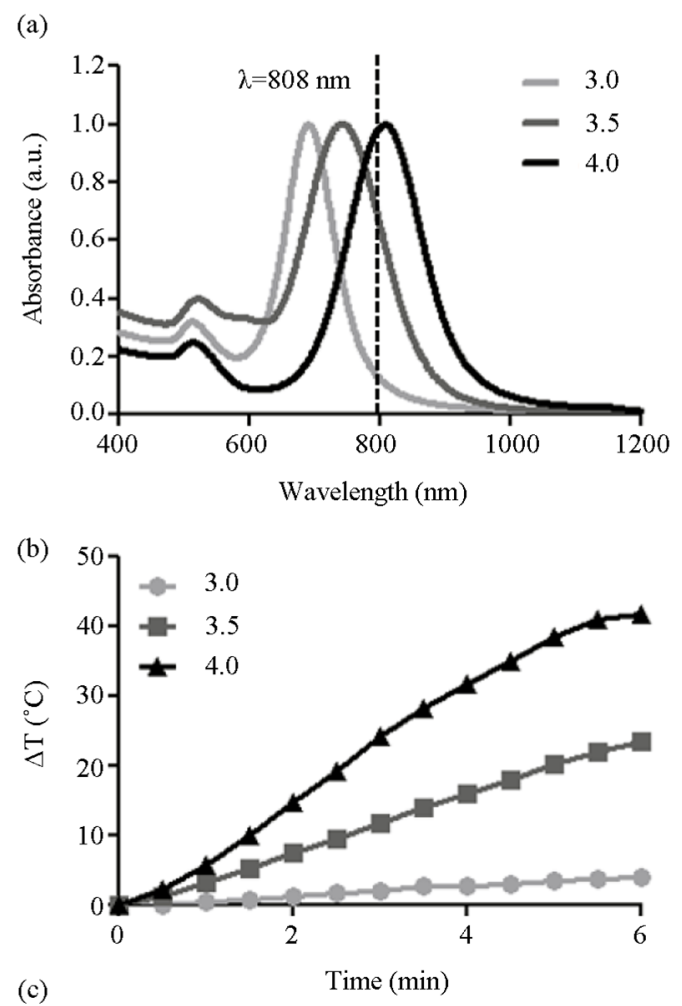

\begin{tabular}{|c|c|c|c|}
\hline $\mathrm{AR}$ & $\mathrm{A}_{808}$ & $\Delta \mathrm{T}(\mathrm{K})$ & $\mathrm{Q}(\mathrm{J} / \mathrm{s})$ \\
\hline 3.0 & 0.11 & 2.4 & 0.16 \\
\hline 3.5 & 0.58 & 13.4 & 0.69 \\
\hline 4.0 & 1.00 & 26.3 & 1.21 \\
\hline
\end{tabular}

Figure 3. Impact of particle absorbance on nanophotonic heating in water. (a) The UV-VIS spectra of Au-NRs with varying AR demonstrate a range of absorbencies at $808 \mathrm{~nm}$, the laser wavelength; (b) The resultant heating profiles of Au-NR solution during NIR laser irradiation identified a direct correlation between photothermal response and absorbance; (c) Results of a basic thermodynamic model which evaluated rate of energy generation for each AR during laser exposure.

face charge, and extent of agglomeration is the composition of the surrounding fluid [13]. Consequently, we predicted that the photothermal capability of Au-NRs would be dependent on the dispersant composition. To test this hypothesis, Au-NRs were distributed in either growth media or an artificial physiological fluid and underwent NIR laser irradiation for six minutes with continual evaluation of solution temperature. Standard cellular growth media served as the control fluid for these experiments and showed no substantial modification in photothermal response compared to water (data not shown).

However, when distributed in the physiological fluids, the Au-NRs exhibited an altered thermal kinetic profile 
for all ARs, as seen in Figure 4. When in alveolar fluid, the nanophotonic response was reduced by approximately $20 \%$, whereas in interstitial and cerebrospinal fluid, the photothermal efficiency dropped by approximately $25 \%$. Similar heating trends were seen for all tested ARs, with the most significant inhibition occurring in interstitial and cerebrospinal fluids. Control experiments verified that the fluids alone produced negligible quantities of heat during NIR exposure (data not shown), confirming that the modified heating response was generated by a fluid-dependent alteration of Au-NR behavior and not the fluids themselves.

\subsection{Modified Au-NR Behavior in Physiological Fluids}

The most probable explanation for this diminution of $\mathrm{Au}-\mathrm{NR}$ photothermal capability is an alteration to the physical behavior of the nanorods; likely an excessive amount of particle agglomeration. Increased agglomeration would result in a reduction of the number of Au-NRs directly in the laser beam path and capable of absorbing the light photons, thus leading to a loss of heat generation. As all the tested ARs displayed an approximate equivalent percent reduction of thermal production in the artificial fluids, it demonstrated that the fluid composition and not the particle AR dominated this effect. As such, only AR 4 Au-NRs were used to evaluate behavior in physio-
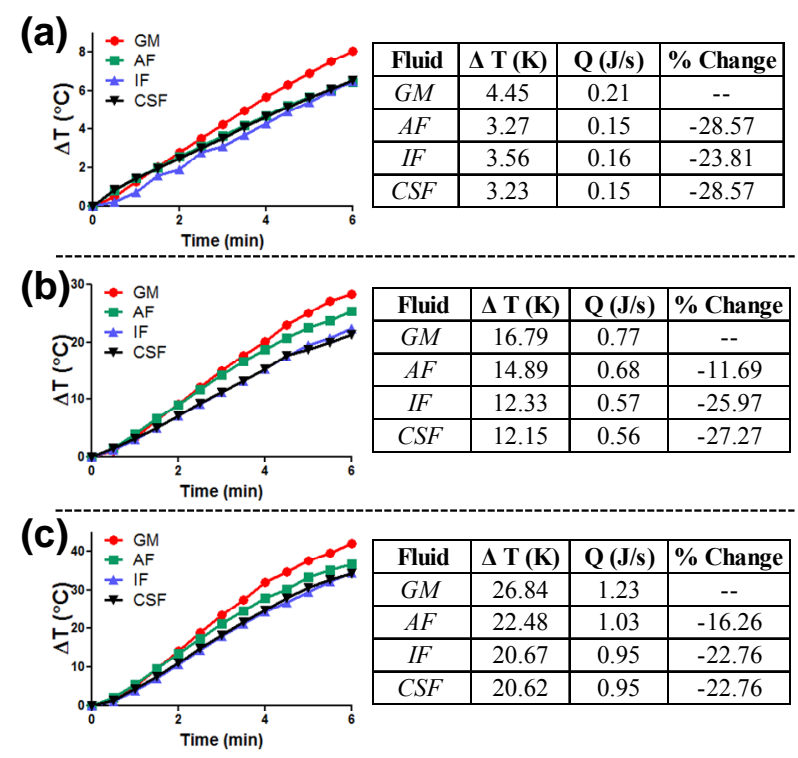

Figure 4. Influence of fluid composition on gold nanorod nanophotonic response. Gold nanorods of AR (a) 3.0, (b), 3.5, and (c) 4.0 were dispersed in either growth media (GM), artificial alveolar fluid (AF), artificial interstitial fluid (IF), or artificial cerebrospinal fluid (CSF) and exposed to an NIR light source. The photothermal response of each particle set was monitored and subjected to the thermodynamic model to ascertain the rate of heat production. logically relevant environments.

As a first metric to evaluate Au-NR behavior in the examined artificial fluids, DLS was performed to quantitatively ascertain the extent of agglomeration (Table 2). These results identified a significant increase in the effective Au-NR size following dispersion in AF, IF, and CSF over GM. Importantly, the agglomerate sizes were inversely proportional to the observed photothermal response, with increasing aggregation correlating to a loss in heating. Of the tested fluids, it was found that the greatest degree of agglomeration and loss of heating were associated with IF and CSF environments. A zeta potential analysis was also performed on each set of $\mathrm{Au}-\mathrm{NRs}$, and the expected alterations to surface charge were found, in accordance with the fluid's composition. To further illuminate the influence of fluid composition on Au-NR behavior UV-VIS analysis and characterization was performed (Figure 5(a), Table 2).

Agglomeration has been shown to induce a number of
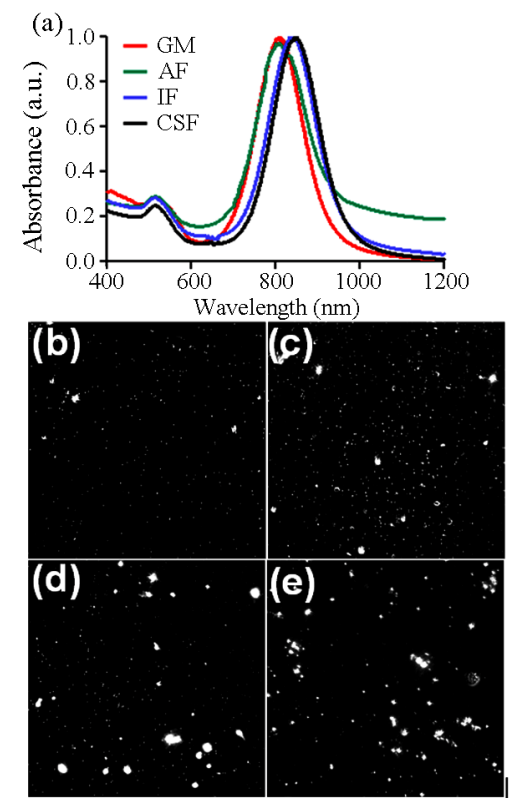

Figure 5. Alteration to gold nanorod behavior in physiological fluids. (a) Fluid dependent alterations in the UV-VIS spectral signatures of gold nanorods. Dark field imaging was performed to visually assess extent of gold nanorod aggregation when in (b) growth media, (c) alveolar fluid, (d) interstitial fluid, and (e) cerebrospinal fluid.

Table 2. Analysis of Au-NR physical parameters in biological fluids.

\begin{tabular}{ccccc}
\hline Fluid & Agglomerate size $(\mathrm{nm})$ & Zeta potential $(\mathrm{mV})$ & $A_{808}$ & $\lambda_{\text {Peak }}$ \\
\hline $\mathrm{GM}$ & $76.6 \pm 1.9$ & $-19.3 \pm 0.7$ & 1.00 & 810 \\
$\mathrm{AF}$ & $162.8 \pm 31.9$ & $-2.9 \pm 1.2$ & 0.95 & 805 \\
$\mathrm{IF}$ & $298.1 \pm 68.5$ & $-6.9 \pm 0.9$ & 0.86 & 841 \\
$\mathrm{CSF}$ & $278.0 \pm 78.4$ & $-6.6 \pm 0.8$ & 0.78 & 850 \\
\hline
\end{tabular}


alterations to the spectral profile of nanorods, including a spectral redshift and LSP peak broadening [24]. Both of these modifications were found when AR 4 Au-NRs were distributed in physiological fluids. In IF and CSF a redshift of 31 and $40 \mathrm{~nm}$, respectively, was noted in the resultant spectral signature, in agreement with DLS agglomeration data. However, when in AF there was no shift to the NIR range, but instead there was a slight broadening of the LSP peak and a diminishment of the maximum absorbance. Included in Table 2 are the $A_{808}$ and $\lambda_{\text {Peak }}$ values for each fluid examined, quantitatively demonstrating fluid-dependent modification of the AuNR spectral profile. Consequently, we concluded that this environmental influence on Au-NR behavior is responsible to for the differential nanophotonic efficiency in physiological fluids (Figure 4).

The examination of fluid-induced particle aggregation was taken one step further to visually assess agglomeration patterns. To achieve this, dark field (DF) imaging was performed with representative images shown in Figures 5(b)-(e). In GM particle are primarily monodispersed with only a few petite aggregates visible. When in $\mathrm{AF}$, there were more agglomerates and the sizes tended to be larger than in GM. However, the most significant alterations were seen in IF and CSF environments; both of which produced exceptionally large $\mathrm{Au}-\mathrm{NR}$ aggregates with a wide range of sizes noted. This variety in aggregates aligns with previous DLS data that indicated a large standard deviation of size when particles were resuspended in IF and CSF.

\subsection{Fluid-Dependent Loss of Nanophotonic Cellular Response}

In addition to quantitatively demonstrating that the photothermal kinetic rate of Au-NRs was strongly dependent on environmental factors, this study sought to ascertain if this modification impacted a targeted cellular response. As one major nanophotonic application to date is the ablation of cells [4], this physiological consequence was selected for evaluation. Subsequently, the ablation potential of AR 4 Au-NRs was evaluated in GM and the three examine artificial physiological fluids. The cells were exposed to a $25 \mu \mathrm{g} / \mathrm{mL}$ concentration, the same as in the acellular evaluation, followed by a one minute irradiation. While all experimental conditions induced a high degree of cellular death, the growth media produced a more robust ablation response when directly compared against the other fluids (Figure 6). Controls without laser exposure were performed to verify that this phenomenon was not an artifact of the fluids themselves, but induced as a result of the joint Au-NR and NIR laser response (data not shown). In growth media, the highest degree of cellular destruction was observed, in excellent agreement with previous results that indicated the smallest degree of

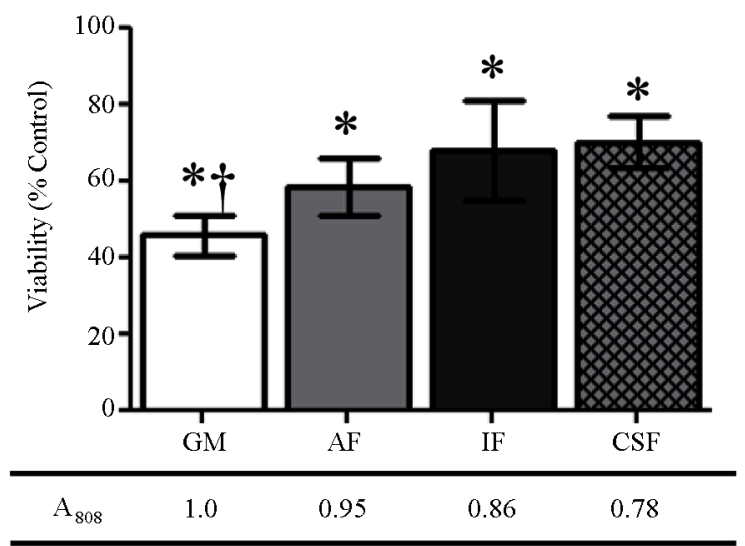

Figure 6. Fluid dependent efficiency of gold nanorod dependent cellular response. The influence of a cellular environment on nanophotonic derived cellular death was evaluated and found to be highly susceptible to environmental composition. The absorbance values for each fluid are included for correlation $\left(^{*}\right.$ and ${ }^{\dagger}$ denote statistical significance from the control and from other fluids, respectively, $\mathrm{p}<$ $0.05, n=4)$.

agglomeration and a more pronounced photothermal effect with GM. The reduction in generated heat in IF and CSF impacted the ability of Au-NRs to destroy targeted $\mathrm{HaCaT}$ cells, demonstrating the potential for environmental interference in nano-based applications.

\subsection{Implications of These Findings for Nano-Based Applications}

The work presented in this study provides knowledge important to consider for both the medical and nanotechnology fields at large. We revealed that the behavior of Au-NRs, both the extent of agglomeration and NIR laser dependent heating efficiency, was modulated in different physiologically-relevant fluids. While it is known that all nanomaterials will display inter-particles interactions to some degree when dispersed in fluids, the extent of that aggregation is dependent upon the fluid composition and ionic strength [13]. The behavior of Au-NRs in interstitial fluid was extremely interesting to explore as it is one of the most prevalent environments in vivo. A high degree of particle agglomeration in IF was identified through DLS, UV-VIS, and DF imaging in this study, suggesting that nanomaterials in an in vivo setting are likely to interact as a group instead of individual rods. A similar effect was previously seen with silver nanoparticles in IF, demonstrating that this is a fluid effect and particle quality concerns [25]. The lesser degree of agglomeration seen in growth media is due to the high serum and protein content of that fluid, which coats the particles, forming a protein corona, and serves as a buffer between the particles, thus minimizing inter-particle forces [26].

Lastly, we demonstrated that the observed fluid-de- 
pendent agglomeration altered the inherent nanophotonic response of Au-NRs. This discovery was validated both acellularly and in an in vitro environment. From these evaluations the degree of agglomeration, extent of photothermal response, and ability of Au-NRs for cellular ablation were all found to be interconnected. Taken together, these results pose an intriguing question as to how nano-sized particles would behave in an in vivo system. As Au-NRs travel through the circulatory system, they would encounter numerous biological locations; each with a different ionic strength and protein concentration and capable of modulating nanorod behavior. As we have demonstrated that Au-NRs agglomeration directly impacts the photothermal response, this phenomenon introduces serious obstructions to nanophotonic reproducibility and efficiency in a physiological environment.

\section{Conclusions}

In this study we quantitatively evaluated the photothermal response of Au-NRs under NIR laser irradiation as both a function of $\mathrm{AR}$ and environmental composition. From AR experimentation, it demonstrated the strong link between nanoparticle absorbance at the operational wavelength $\left(\mathrm{A}_{808}\right)$ and the kinetic heating rate.

This work also identified that in physiological fluids, the Au-NRs agglomerated to a large degree. Moreover, this aggregration was determined to be more significant than in traditional cell culture media, indicating modified $\mathrm{Au}-\mathrm{NR}$ behavior. Fluid dependent alterations to particle behavior induced a shift in the spectral profile, produced a reduction in the $A_{808}$ value, and resulted in a decrease in nanophotonic efficiency. This diminution of energy production reduced the targeted cellular ablation initiated through a joint nanomaterial-laser induced mechanism. These findings are pictorially summarized in Figure 7. This study enhanced fundamental knowledge and under-

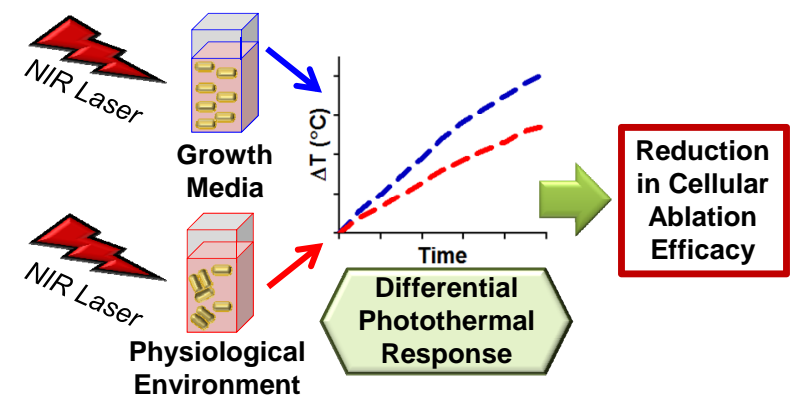

Figure 7. Schematic summary of the gold nanorod photothermal response following dispersion in alternative physiological fluids. Dispersant composition induced either a low or high degree of agglomeration, which impacted the absorbance and the dependent nanophotonic thermal generation. This decrease in photothermal effect resulted in a loss of target application efficiency. standing of Au-NR behavior in a physiological environment and during NIR laser irradiation, a combination that is becoming increasingly common in medical research and applications. Therefore, to progress NIR-based in vivo applications in the biotechnology and nanotechnology sectors, future studies must address the localized environmental factors that may potentially impact nanomaterial association and behavior.

\section{Acknowledgements}

The authors would like to thank Ms. Elizabeth Maurer for TEM imaging of the Au-NRs and Ms. Emily Breitner for assistance with DF imaging. Portions of this work were funded through the 711th HPW/AFRL Chief Scientist Seedling Program, Tier 1 and the Air Force Surgeon General. K.K.C. was supported through a National Research Council post-doctoral fellowship, funded through the Air Force Office of Scientific Research. B.M.S. received funding from the Oak Ridge Institute for Science and Education.

\section{REFERENCES}

[1] M. C. Daniel and D. Astruc, "Gold Nanoparticles: Assembly, Supramolecular Chemistry, Quantum-Size-Related Properties, and Applications Toward Biology, Catalysis, and Nanotechnology," Chemical Reviews, Vol. 104, No. 1, 2004, pp. 293-346. http://dx.doi.org/10.1021/cr030698+

[2] L. Dykman and N. Khlebtsov, "Gold Nanoparticles in Biomedical Applications: Recent Advances and Perspectives," Chemical Society Reviews, Vol. 41, No. 6, 2012, pp. 2256-2282. http://dx.doi.org/10.1039/c1cs15166e

[3] Y. Wang, X. Xie, X. Wang, G. Ku, K. L. Gill, D. P. O'Neal, G. Stoica and L. V. Wang, "Photoacoustic Tomography of a Nanoshell Contrast Agent in the in Vivo Rat Brain," Nano Letters, Vol. 4, No. 9, 2004, pp. 16891692. http://dx.doi.org/10.1021/n1049126a

[4] E. C. Dreaden, A. M. Alkilany, X. Huang, C. J. Murphy and M. A. El-Sayed, "The Golden Age: Gold Nanoparticles for Biomedicine," Chemical Society Reviews, Vol. 41, No. 7, 2012, pp. 2740-2779. http://dx.doi.org/10.1039/c1cs15237h

[5] X. Huang, I. H. El-Sayed, W. Qian and M. A. El-Sayed, "Cancer Cell Imaging and Photothermal Therapy in the Near-Infrared Region by Using Gold Nanorods," Journal of the American Chemical Society, Vol. 128, No. 6, 2006, pp. 2115-2120. http://dx.doi.org/10.1021/ja057254a

[6] H. H. Richardson, M. T. Carlson, P. J. Tandler, P. Hernandez and A. O. Govorov, "Experimental and Theoretical Studies of Light-to-Heat Conversion and Collective Heating Effects in Metal Nanoparticle Solutions," Nano Letters, Vol. 9, No. 3, pp. 1139-1146. http://dx.doi.org/10.1021/n18036905

[7] A. M. Alkilany, L. B. Thompson, S. P. Boulos, P. N. Sisco and C. J. Murphy, "Gold Nanorods: Their Potential for Photothermal Therapeutics and Drug Delivery, Tem- 
pered by the Complexity of Their Biological Interactions," Advanced Drug Delivery Reviews, Vol. 64, No. 2, 2012, pp. 190-199. http://dx.doi.org/10.1016/j.addr.2011.03.005

[8] W. I. Choi, J. Kim, C. Kang, C. C. Byeon, Y. H. Kim and G. Tae, "Tumor Regression in Vivo by Photothermal Therapy Based on Gold-Nanorod-Loaded, Functional Nanocarriers," ACS Nano, Vol. 5, No. 3, 2011, pp. 19952003. http://dx.doi.org/10.1021/nn103047r

[9] T. S. Hauck, T. L. Jennings, T. Yatsenko, J. C. Kumaradas and W. C. W. Chan, "Enhancing the Toxicity of Cancer Chemotherapeutics with Gold Nanorod Hyperthermia," Advanced Materials, Vol. 20, No. 20, 2008, pp. 3832-3838. http://dx.doi.org/10.1002/adma.200800921

[10] J. Huang, K. S. Jackson and C. J. Murphy, "Polyelectrolyte Wrapping Layers Control Rates of Photothermal Molecular Release from Gold Nanorods," Nano Letters, Vol. 12, No. 6, 2012, pp. 2982-2987. http://dx.doi.org/10.1021/nl3007402

[11] S. Link, M. B. Mohamed and M. A, El-Sayed, "Simulation of the Optical Absorption Spectra of Gold Nanorods as a Function of their Aspect Ratio and the Effect of the Medium Dielectric Constant," Journal of Physical Chemistry B, Vol. 103, No. 16, 1999, pp. 3073-3077. http://dx.doi.org/10.1021/jp990183f

[12] J. C. Y. Kah, A. Zubieta, R. A. Saavedra and K. HamadSchifferli, "Stability of Gold Nanorods Passivated with Amphiphilic Ligands," Langmuir, Vol. 28, No. 24, 2012, pp. 8834-8844. http://dx.doi.org/10.1021/la3000944

[13] M. Sethi, G. Joung and M. R. Knecht, "Stability and Electrostatic Assembly of Au Nanorods for Use in Biological Assays," Langmuir, Vol. 25, No. 1, pp. 317-325. http://dx.doi.org/10.1021/la802096v

[14] P. K. Jain, K. S. Lee, I. H. El-Sayed and M. A. El-Sayed, "Calculated Absorption and Scattering Properties of Gold Nanoparticles of Different Size, Shape, and Composition: Applications in Biological Imaging and Biomedicine," Journal of Physical Chemistry B, Vol. 110, No. 14, 2006, pp. 7238-7248. http://dx.doi.org/10.1021/jp057170o

[15] B. M. Stacy, K. K. Comfort, D. A. Comfort and S. M. Hussain, "In Vitro Identification of Gold Nanorods through Hyperspectral Imaging," Plasmonics, Vol. 8, No. 2, 2013, pp. 1235-1240. http://dx.doi.org/10.1007/s11468-013-9538-6

[16] K. K. Comfort, E. I. Maurer, L. K. Braydich-Stolle and S. M. Hussain, "Interference of Silver, Gold, and Iron Oxide Nanoparticles on Epidermal Growth Factor Signal Transduction in Epithelial Cells," ACS Nano, Vol. 5, No. 12, 2011, pp. 10000-10008. http://dx.doi.org/10.1021/nn203785a

[17] M. C. DeBrosse, K. K. Comfort, E. A. Untener, D. A. Comfort and S. M. Hussain, "High Aspect Ratio Gold Nanorods Displayed Augmented Cellular Internalization and Surface Chemistry Mediated Cytotoxicity," Materials Science and Engineering C, Vol. 33, No. 7, 2013, pp. 4094-4100. http://dx.doi.org/10.1016/j.msec.2013.05.056

[18] K. Park and R. A. Vaia, "Synthesis of Complex Au/Ag Nanorods by Controlled Overgrowth," Advanced Materials, Vol. 20, No. 20, 2008, pp. 3882-3886. http://dx.doi.org/10.1002/adma.200800613

[19] K. Park, H. Koerner and R. A. Vaia, "Depletion-Induced Shape and Size Selection of Gold Nanoparticles," Nano Letters, Vol. 10, No. 4, 2010, pp. 1433-1439. http://dx.doi.org/10.1021/nl100345u

[20] Y, Liu, M. K. Shipton, J. Ryan, E. D. Kaufman, S. Frazen and D. L. Feldheim, "Synthesis, Stability, and Cellular Internalization of Gold Nanoparticles Containing Mixed Peptide-Poly(ethylene glycol) Monolayers," Analytical Chemistry, Vol. 79, No. 6, 2007, pp. 2221-2229. http://dx.doi.org/10.1021/ac061578f

[21] W. Stopford, J. Turner, D. Cappellini and T. Brock, "Bioaccessibility Testing of Cobalt Compounds," Journal of Environmental Monitoring, Vol. 5, No. 4, 2003, pp. 675680. http://dx.doi.org/10.1039/b302257a

[22] J. Alper and K. Hamad-Schifferli, "Effect of Ligand on Thermal Dissipation from Gold Nanorods," Langmuir, Vol. 26, No. 6, 2010, pp. 3786-3789. http://dx.doi.org/10.1021/la904855s

[23] A. M. Alkilany, P. K. Nagaria, C. R. Hexel, T. J. Shaw, C. J. Murphy and M. D. Wyatt, "Cellular Uptake and Cytotoxicity of Gold Nanorods: Molecular Origin of Cytotoxicity and Surface Effects," Small, Vol. 5, No. 6, 2009, pp. 701-708. http://dx.doi.org/10.1002/smll.200801546

[24] V. P. Pattani and J. W. Tunnell, "Nanoparticle-Mediated Photothermal Therapy: A Comparative Study of Heating for Different Particle Types," Lasers in Surgery and Medicine, Vol. 44, No. 8, 2012, pp. 675-684. http://dx.doi.org/10.1002/1sm.22072

[25] L. V. Stebounova, E. Guio and V. H. Grassian, "Silver Nanoparticles in Simulated Biological Media: A Study of Aggregation, Sedimentation, and Dissolution," Journal of Nanoparticle Research, Vol. 13, No. 1, 2011, pp. 233-244. http://dx.doi.org/10.1007/s11051-010-0022-3

[26] I. Lynch, A. Salvati and K. A. Dawson, "Protein-Nanoparticle Interactions: What Does the Cell See?" Nature Nanotechnology, Vol. 4, No. 9, 2009, pp. 546-547. http://dx.doi.org/10.1038/nnano.2009.248 